ORIGINAL

\title{
PERFIL CLÍNICO-EPIDEMIOLÓGICO \\ DE PACIENTES QUE INICIAN TERAPIA INTENSIVA CON ESTATINAS PARA LA PREVENCIÓN SECUNDARIA DE ENFERMEDAD VASCULAR EN ESPAÑA
}

\author{
Diego Macías Saint-Gerons (1), César de la Fuente Honrubia (1), Diana González Bermejo (1), \\ Dolores Montero (1), Miguel J Gil (1), Antonio Salvador Sanz (2), Fernando de Andrés-Trelles (3) \\ y Ferrán Catalá-López (1).
}

(1) División de Farmacoepidemiología y Farmacovigilancia. Agencia Española de Medicamentos y Productos Sanitarios (AEMPS). Madrid. España.

(2) Servicio de Cardiología, Hospital Universitario y Politécnico La Fe. Valencia. España.

(3) Departamento de Farmacología. Facultad de Medicina. Universidad Complutense de Madrid. España.

Los autores declaran no tener conflictos de interés.

Los datos para la realización de este trabajo son parte de la base de datos BIFAP gestionada por la Agencia Española de Medicamentos y Productos Sanitarios (AEMPS). Los resultados, discusión y conclusiones de este estudio no representan en ningún modo la posición de la AEMPS respecto a este tema

\section{RESUMEN}

Fundamentos: Las nuevas recomendaciones respecto a la utilización de estatinas potentes y/o dosis altas (terapia intensiva) para el tratamiento de la enfermedad cardiovascular se han basado en la extrapolación de los resultados de los ensayos clínicos. El objetivo fue describir el perfil clínicoepidemiológico de los pacientes que inician tratamiento con estatinas para la prevención secundaria en España y estudiar qué factores determinan la utilización de estatinas en terapia intensiva.

Métodos: Estudio transversal a partir de 88.751 pacientes $\geq 45$ años con enfermedad cardiovascular que iniciaron tratamiento con estatinas (enero 2007-diciembre 2011). Los tratamientos con dosis superiores a $40 \mathrm{mg} / \mathrm{dí}$ de simvastatina (o estatina equipotente) se consideraron terapia intensiva. Se construyeron modelos de regresión logística multivariante con el fin de examinar la asociación de las variables relacionadas con la prescripción de terapia intensiva respecto al uso de la terapia moderada.

Resultados: Se identificó a 16.857 personas adultas que iniciaron tratamiento con estatinas para la prevención secundaria. Los factores predictores para el inicio de terapia intensiva fueron el año de prescripción, sexo masculino (odds ratio ajustada: 1,70; IC95\%: 1,44-2,00), edad $>75$ años $(1,39 ; 1,15-1,69)$, historia previa de enfermedad isquémica coronaria $(1,71$; $1,44-2,04)$, accidente isquémico transitorio de cualquier localización $(1,24$ $0,97-1,59)$, tabaquismo $(1,62 ; 1,34-1,95)$, hipertensión $(1,41 ; 1,20-1,65)$ y tratamiento reciente con fibratos $(2,32 ; 1,27-4,26)$.

Conclusiones: La utilización de terapia intensiva con estatinas está determinada por el tipo de evento vascular previo y con la edad ( $>75$ años, en los que el balance beneficio-riesgo podría ser discutible). No se encuentran diferencias estadísticamente significativas en función del c-LDL.

Palabras clave: Farmacoepidemiología. Utilización de medicamentos. Inhibidores de la HMG-CoA reductasa, Dosificación. España.

Correspondencia

Diego Macías Saint-Gerons

División de Farmacoepidemiología y Farmacovigilancia

Agencia Española de Medicamentos y Productos Sanitarios (AEMPS)

Calle Campezo 1, Edificio 8, E-28022 Madrid, España/Spain

dmacias.sg@gmail.com

DOI:

\section{ABSTRACT \\ Clinical-Epidemiological Profile of Patients Initiating Intensive Statin Therapy for the Secondary Prevention of Vascular Disease in Spain}

Background: The new recommendations regarding the utilization of high potency statins (intensive therapy) for the treatment of cardiovascular disease have been based on the extrapolation of data coming from clinical trials. The objective is to describe the clinical-epidemiological profile of statin therapy users for the secondary prevention of cardiovascular disease in Spain and to examine the predictors for intensive therapy initiation.

Methods: Cross-sectional study from a sample of 88,751 patients aged $\geq 45$ years-old with previous cardiovascular disease which initiated statin therapy between 1 st January 2007 to 31 st December 2011. Dose treatments $>40 \mathrm{mg}$ simvastatin daily (or equivalent dose if different statin) were considered intensive therapy treatment. Multivariable logistic regression models were built for dependent summary variables to examine the association between and the intensive therapy utilization (vs low-moderate intensity therapy).

Results: 16,857 adult patients receiving a first prescription of statin for the secondary prevention of cardiovascular diseases were identified. Predictors for intensive therapy initiation were year of statin prescription, male gender (adjusted OR: $1.70 ; 95 \% \mathrm{CI}: 1.44-2.00)$, age $>75$ years-old $(1.39$; $1.15-1.69)$, previous history of coronary artery disease $(1.71 ; 1.44-2.04)$, previous history of transient ischemic attack (1.24;0,97-1.59), smoking (1.62; 1.34-1.95), hypertension $(1.41 ; 1.20-1.65)$ and recent use of fibrates $(2.32 ; 1.27-4.26)$.

Conclusions: The onset of intensive therapy with statins in secondary was determined by the type of vascular event and age ( $>75$ years-old in which the risk benefit balance could be controversial). No statistically significant differences were found according to the LDL-c levels

Keywords: Pharmacoepidemiology, Drug utilization, HMG-CoA reductase inhibitors, dosage, Spain. 


\section{INTRODUCCIÓN}

Las estatinas son un grupo de fármacos que han demostrado su eficacia en la prevención secundaria de la enfermedad cardiovascular ${ }^{1}$. Además, la introducción de genéricos junto con la disminución de los precios ${ }^{2}$ han contribuido al aumento del consumo de estatinas en la última década ${ }^{3}$.

La estrategia terapéutica más agresiva de utilización de estatinas (terapia intensiva) consiste en el uso de nuevas estatinas dotadas de mayor capacidad para la reducción de los niveles de colesterol unido a lipoproteinas de baja densidad (cLDL) y el uso de dosis altas. Este abordaje terapéutico podría haber aumentado desde la publicación de ensayos clínicos como el estudio Treating to New Targets study (TNT) $)^{4}$, en el que se concluye que la atorvastatina de $80 \mathrm{mg}$ ofrece beneficios adicionales en la reducción de eventos cardiovascularesrespecto a la atorvastatina de 10 $\mathrm{mg}^{4}$. Por otra parte, en la reciente revisión de las guías de práctica clínica europeas y americanas, se han incluido recomendaciones con énfasis en la utilización de la terapia intensiva con estatinas ${ }^{5-7}$, aunque también existe preocupación por el posible aumento del riesgo de reacciones adversas con la terapia intensiva ${ }^{8,9}$ que podría ser mayor en presencia de contraindicaciones o interacciones ${ }^{10}$.

Sin embargo, para la prevención secundaria son poco conocidos hasta la fecha los factores que determinan la utilización de terapia intensiva con estatinas -dosis más altas y/o estatinas más potentes- en lugar del uso de terapia de intensidad moderada con estatinas.

El objetivo principal del presente estudio de base poblacional fue conocer el perfil clínico y epidemiológico de las personas con historia previa de enfermedad cardiaca y/o vascular tratados con estatinas en España y explorar las variables que se asocian con la utilización de estatinas potentes y/o dosis altas (terapia intensiva) respecto al uso de una estrategia de tratamiento con estatinas menos agresiva pero más segura (terapia moderada), así como la presencia de posibles contraindicaciones o interacciones medicamentosas, reflejando el uso en condiciones de práctica clínica habitual.

\section{SUJETOS Y MÉTODO}

Diseño del estudio y participantes. Se llevó a cabo un análisis transversal utilizando datos individuales procedentes de la Base de datos para la Investigación Farmacoepidemiológica en Atención Primaria (BIFAP), la cual es una base de datos poblacional longitudinal financiada por la Agencia Española de Medicamentos y Productos Sanitarios (AEMPS) que, desde 2001 en adelante, contiene historias clínicas informatizadas de más de 4 millones de pacientes atendidos por más de 2.200 especialistas de atención primaria en España. La información disponible de manera anonimizada representa 20 millones de personas-año de seguimiento ${ }^{11}$.

Se incluyó a todas las personas de ambos sexos mayores de 45 años, con historia previa de cardiopatía isquémica, infarto agudo de miocardio (IAM), accidente cerebrovascular (ACV), accidente isquémico transitorio (AIT) o enfermedad arterial periférica (de acuerdo a la Clasificación Internacional de Atención Primaria (CIAP), con la primera prescripción de estatina entre el 1 de enero de 2007 y el 31 de diciembre de 2011 y que tuvieran al menos un año de seguimiento con su médico.

La fecha de la primera prescripción de estatina fue considerada como "fecha índice" del estudio (index date). Los pacientes se definieron como iniciadores de terapia moderada con estatinas si no tenían registrada la prescripción de ninguna estatina previa a la fecha índice. Por otra parte, se definió a los iniciadores de terapia intensiva con estatinas a los pacientes con una primera prescripción de estatina en terapia intensiva sin registro previo de ninguna estatina o bien si habían recibido una única prescripción a dosis moderada seguida de una segunda prescripción a dosis alta (titulación de dosis) y ninguna estatina previa a la fecha índice. 
Clasificación de la exposición y covariables. A partir de la base de datos se obtuvo el uso de estatinas en la fecha índice [definidas según códigos del sistema de clasificación Anatómica, Terapéutica, Química (ATC)]. La clasificación de la exposición se hizo de forma similar a otros estudios ${ }^{11,12}$. Se consideró como expuestos a terapia intensiva a los sujetos con una dosis prescrita de simvastatina superior a $40 \mathrm{mg} /$ día o con una dosis diaria de otra estatina con capacidad equivalente para la reducción de los niveles plasmáticos de cLDL (atorvastatina $\geq 40 \mathrm{mg}$, fluvastatina $>80 \mathrm{mg}$, lovastatina $>80 \mathrm{mg}$, pitavastatina $>4 \mathrm{mg}$, pravastatina $>80 \mathrm{mg}$, rosuvastatina $>5 \mathrm{mg})^{7,13}$.

A partir de la historia clínica informatizada se obtuvieron las características sociodemográficas y clínicas de los sujetos, incluyendo edad, sexo, índice de masa corporal en $\mathrm{kg}$ / $\mathrm{m}^{2}$ (IMC), presencia de comorbilidades crónicas relevantes (utilizando códigos CIAP, o comentarios de texto en la historia clínica), registradas como diabetes mellitus, hipotiroidismo, enfermedad renal (insuficiencia renal y/o proteinuria), hipertensión, hábito tabáquico e ingesta elevada de alcohol.

También se obtuvieron los valores lipídicos registrados hasta el mes previo a la fecha índice y se descartaron aquellos valores con una fecha registrada de 12 meses anterior a la fecha índice.

Se consideró colesterol elevado cuando el colesterol total (CT) era superior a $200 \mathrm{mg} /$ $\mathrm{d}^{11,14}$; triglicéridos elevados si su valor era igual o mayor a150 mg/dl ${ }^{15}$; bajo nivel de colesterol unido a lipoproteinas de alta densidad (cHDL) cuando los valores eran inferiores a $40 \mathrm{mg} /$ $\mathrm{dl}$ en hombres y $50 \mathrm{mg} / \mathrm{dl}$ en mujeres ${ }^{14,16}$. Los niveles de cLDL se clasificaron en categorías según criterios para el inicio de tratamiento con estatinas: $<130 \mathrm{mg} / \mathrm{dl}, 130-159,9 \mathrm{mg} / \mathrm{dl}$, $160-189,9 \mathrm{mg} / \mathrm{dl}, \mathrm{y} \geq 190 \mathrm{mg} / \mathrm{dl}^{16}$.

Se obtuvo información de la medicación relevante que consumían los participantes, incluyendo diuréticos, antidiabéticos, vaso- dilatadores, beta bloqueantes, inhibidores del sistema renina angiotensina y tiroxina. El uso previo de hipolipemiantes (fibratos, ezetimiba y otros como secuestrantes de ácidos biliares, omega-3, ácido nicotínico/ laropiprant) fue clasificado como "uso actual" cuando existía una prescripción 30 días antes de la fecha índice, "uso reciente" con prescripción 30-60 días antes de la fecha índice y "uso pasado/no uso" en caso de prescripción con antigüedad mayor a 60 días o sin ninguna prescripción registrada.

También se obtuvo información de medicamentos que pudiesen interaccionar con las estatinas, teniendo en cuenta la vía metabólica de esta última (por ejemplo antagonistas del calcio, inductores del citocromo CYP34A4, inhibidores del citocromo CYP3A4 y ciclosporina) $)^{17,18}$.

Análisis estadístico. Las características de los participantes se describieron utilizando proporciones. Además se construyeron modelos de regresión logística para las variables dependientes con el fin de evaluar su fuerza de asociación con la utilización de terapia intensiva con estatinas. Uno principal (modelo A) con los valores de lípidos de laboratorio y otro (modelo B) sin valores lipídicos. Se obtuvieron odds ratios (OR) con su correspondiente intervalo de confianza del 95\% (IC95\%) para las distintas covariables utilizando el método de eliminación retrógrada (backward elimination), con una probabilidad de eliminación del 0,10 y una probabilidad de entrada para las variables significativas de 0,05. La bondad de ajuste se evaluó utilizando el test de Hosmer-Lemeshow y la capacidad de discriminación mediante el estadístico C. Todos los análisis se llevaron a cabo utilizando el programa estadístico Stata 12.1 (Stata Corp, College Station, TX).

\section{RESULTADOS}

Se identificó a un total de 88.751 sujetos con una primera prescripción de estatina. Tras excluir a los pacientes en tratamiento para prevención primaria, se incluyó a 16.857 personas con 


\section{Figura 1 \\ Diagrama de flujo del estudio}

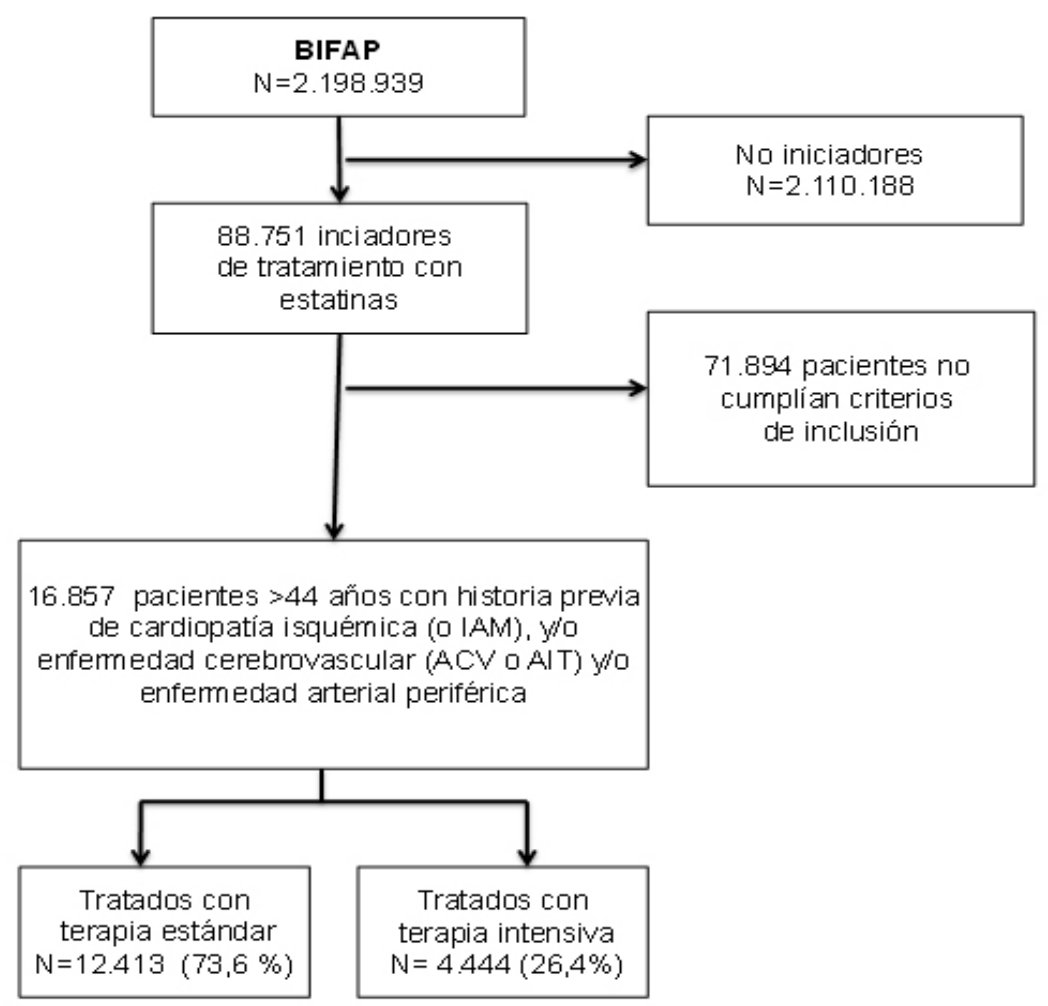

BIFAP: Base de datos para la Investigación Farmacoepidemiológica en Atención Primaria

edad mayor o igual a 45 años, con antecedentes previos de enfermedad vascular o cardiovascular (figura 1). Las principales características de los pacientes se muestran en la tabla 1 .

La edad media de los pacientes fue de 67,9 años y el 61,3\% eran hombres, el 54,4\% tenía historia previa de cardiopatía isquémica, el $33,9 \%$ de accidente cerebro vascular (ACV), el 13,0\% de AIT, 13,2\% enfermedad arterial periférica y el 5,4\% presentaba adicionalmente insuficiencia cardiaca. Una gran proporción de los pacientes tenían factores de riesgo adicio- nal, como CT elevado (70,3\%), hipertensión arterial tratada $(45,2 \%)$, sobrepeso/obesidad leve $(33,6 \%)$, tabaquismo $(27,1 \%)$, consumo elevado de alcohol $(7,4 \%)$ o hipotiroidismo $(6,0 \%)$. Eran iniciadores de terapia moderada 12.413 $(73,6 \%)$ y $4.444(26,4 \%)$ de terapia intensiva. Entre los primeros, las estatinas más utilizadas en el periodo de estudio fueron simvastatina (58,5\% de los pacientes), atorvastatina $(27,1 \%)$ y pravastatina $(7,3 \%)$. Entre los iniciadores de terapia intensiva, las estatinas más utilizadas fueron atorvastatina $(91,3 \%)$ y rosuvastatina $(6,2 \%)$ (tablas 2 y 3$)$. 


\begin{tabular}{|c|c|c|c|}
\hline \multicolumn{4}{|c|}{$\begin{array}{c}\text { Tabla } 1 \\
\text { Características basales de los pacientes tratados con estatinas } \\
\text { en prevención secundaria }\end{array}$} \\
\hline & $\begin{array}{l}\text { Iniciadores } \\
\text { con terapia } \\
\text { moderada } \\
\mathrm{n}=12.413(\%)\end{array}$ & $\begin{array}{l}\text { Iniciadores } \\
\text { con terapia } \\
\text { intensiva } \\
\mathrm{n}=4.444(\%)\end{array}$ & $\begin{array}{c}\text { Total } \\
\text { iniciadores } \\
\mathrm{n}=16.857(\%)\end{array}$ \\
\hline \multicolumn{4}{|l|}{ Edad (años) } \\
\hline $45-64$ & $4.838(38,98)$ & $2.005(45,12)$ & $6.843(40,59)$ \\
\hline $65-74$ & $3.421(27,56)$ & $1.037(23,33)$ & $4.458(26,45)$ \\
\hline $75-101$ & $4.154(33,46)$ & $1.402(31,55)$ & $5.556(32,96)$ \\
\hline \multicolumn{4}{|l|}{ Sexo } \\
\hline Mujeres & $5.216(42,02)$ & $1.310(29,48)$ & $6.526(38,71)$ \\
\hline Hombres & $7.197(57,98)$ & $3.134(70,52)$ & $10.331(61,29)$ \\
\hline \multicolumn{4}{|l|}{ Año } \\
\hline 2007 & $3.570(28,76)$ & $924(20,79)$ & $4.494(26,66)$ \\
\hline 2008 & $3.100(24,97)$ & $981(22,07)$ & $4.081(24,21)$ \\
\hline 2009 & $2.724(21,94)$ & $1.012(22,77)$ & $3.736(22,16)$ \\
\hline 2010 & $1.880(15,15)$ & $965(21,71)$ & $2.845(16,88)$ \\
\hline 2011 & $1.139(9,18)$ & $562(12,65)$ & $1.701(10,09)$ \\
\hline \multicolumn{4}{|l|}{ IMC (kg/m2) } \\
\hline$\leq 25$ & $1.756(18,73)$ & $544(18,19)$ & $2.300(18,60)$ \\
\hline $25,1-29,9$ & $4.264(45,49)$ & $1.365(45,65)$ & $5.629(45,53)$ \\
\hline $30-39,9$ & $3.131(33,40)$ & $1.027(34,35)$ & $4.158(33,63)$ \\
\hline$\geq 40$ & $222(2,37)$ & $54(1,81)$ & $276(2,23)$ \\
\hline N utilizada b (\%) & $9373(75,51)$ & $2.990(67,8)$ & $12.363(73,34)$ \\
\hline \multicolumn{4}{|l|}{ Tabaquismo } \\
\hline Fumador & $2.353(24,85)$ & $1.065(34,07)$ & $3.418(27,14)$ \\
\hline N utilizada b (\%) & $9.468(76,28)$ & $3.126(70.34)$ & $12.594(74,71)$ \\
\hline Consumo elevado de alcohol & $875(7,05)$ & $364(8,19)$ & $1.239(7,35)$ \\
\hline Enfermedad isquémica coronaria & $6.500(52,36)$ & $2.676(60,22)$ & $9.176(54,43)$ \\
\hline Insuficiencia cardiaca & $709(5,71)$ & $206(4,64)$ & $915(5,43)$ \\
\hline Accidente cerebrovascular & $4.243(34,18)$ & $1.465(32,97)$ & $5.708(33,86)$ \\
\hline Accidente isquémico transitorio & $1.624(13,08)$ & $563(12,67)$ & $2.187(12,97)$ \\
\hline Enfermedad arterial periférica & $1.807(14,56)$ & $419(9,43)$ & $2.226(13,21)$ \\
\hline Hipertensión & $7.859(63,31)$ & $2.495(56,14)$ & $10.354(61,42)$ \\
\hline Tratada & $5.612(45,21)$ & $2.002(45,05)$ & $7.614(45,17)$ \\
\hline Diabetes mellitus & $4.091(32,96)$ & $1.149(25,86)$ & $5.240(31,09)$ \\
\hline Tratada & $2.565(20,66)$ & $828(18,63)$ & $3.393(20,13)$ \\
\hline Hipotiroidismo $^{\mathrm{a}}$ & $810(6,53)$ & $204(4,59)$ & $1.014(6,02)$ \\
\hline Enfermedad renal & $698(5,62)$ & $193(4,34)$ & $891(5,29)$ \\
\hline Proteinuria & $156(1,26)$ & $45(1,01)$ & $201(1,19)$ \\
\hline CT elevado & $3.487(71,84)$ & $977(65,39)$ & $4.464(70,32)$ \\
\hline $\mathrm{N}$ utilizada b $(\%)$ & $4.854(39,10)$ & $1.494(33,62)$ & $6.348(37,66)$ \\
\hline \multicolumn{4}{|l|}{$\operatorname{cLDL}(\mathrm{mg} / \mathrm{dl})$} \\
\hline$<130$ & $1.501(34,29)$ & $544(38,91)$ & $2.045(35,41)$ \\
\hline $130-159,9$ & $1.588(36,28)$ & $486(34,76)$ & $2.074(35,91)$ \\
\hline $160-189,9$ & $950(21,70)$ & $299(21,39)$ & $1.249(21,63)$ \\
\hline$\geq 190$ & $338(7,72)$ & $69(4,94)$ & $407(7,05)$ \\
\hline N utilizada b (\%) & $4.377(35,26)$ & $1.398(31,46)$ & $5.775(34,26)$ \\
\hline Triglicéridos elevados & $1,506(32,47)$ & $464(32,81)$ & $1.970(32,55)$ \\
\hline N utilizada b (\%) & $4.638(37,36)$ & $1.414(31,82)$ & $6.052(35,90)$ \\
\hline Bajo cHDL & $1.353(29,05)$ & $457(31,37)$ & $1.810(29,60)$ \\
\hline $\mathrm{N}$ utilizada b (\%) & $4.657(37,52)$ & $1.457(32,79)$ & $6.114(36,27)$ \\
\hline \multicolumn{4}{|l|}{ Medicación cardiovascular } \\
\hline ACE inh/ARBs & $6.685(53,85)$ & $2.782(62,60)$ & $9.467(56,16)$ \\
\hline Antiagregantes & $8.021(64,62)$ & $3.828(86,14)$ & $11.849(70,29)$ \\
\hline Beta bloqueantes & $3.708(29,87)$ & $1.936(43,56)$ & $5.644(33,48)$ \\
\hline Diuréticos & $3.345(26,95)$ & $1.047(23,56)$ & $4.392(26,05)$ \\
\hline Vasodilatadores & $481(3,87)$ & $163(3,67)$ & $644(3,82)$ \\
\hline \multicolumn{4}{|l|}{ Antidiabéticos } \\
\hline Insulina & $674(5,43)$ & $222(5,00)$ & $896(5,32)$ \\
\hline Antidiabéticos orales & $2.368(19,08)$ & $784(17,64)$ & $3.152(18,70)$ \\
\hline \multicolumn{4}{|l|}{ Hipolipemiantes } \\
\hline \multicolumn{4}{|l|}{ Fibratos } \\
\hline Uso concomitante & $230(1,85)$ & $95(2,14)$ & $325(1,93)$ \\
\hline Uso reciente & $84(0,68)$ & $37(0,83)$ & $121(0,72)$ \\
\hline Uso pasado/no uso & $12.099(97,47)$ & $4.312(97,03)$ & $16.411(97,35)$ \\
\hline \multicolumn{4}{|l|}{ Ezetimiba } \\
\hline Uso concomitante & $69(0,56)$ & $43(0,97)$ & $112(0,66)$ \\
\hline Uso reciente & $11(0,09)$ & $1(0,02)$ & $12(0,07)$ \\
\hline Uso pasado/no uso & $12.333(99,36)$ & $4.400(99,01)$ & $16.733(99,26)$ \\
\hline
\end{tabular}




\begin{tabular}{|c|c|c|c|}
\hline \multicolumn{4}{|c|}{$\begin{array}{c}\text { Tabla } 1 \\
\text { continuación }\end{array}$} \\
\hline & $\begin{array}{c}\text { Iniciadores } \\
\text { con terapia } \\
\text { moderada } \\
n=12.413\end{array}$ & $\begin{array}{c}\text { Iniciadores } \\
\text { con terapia } \\
\text { intensiva } \\
n=4.444\end{array}$ & $\begin{array}{c}\text { Total } \\
\text { iniciadores } \\
\mathrm{n}=16.857\end{array}$ \\
\hline \multicolumn{4}{|l|}{ Otros hipolipemiantes } \\
\hline Uso concomitante & $54(0,44)$ & $27(0,61)$ & $81(0,48)$ \\
\hline Uso reciente & $10(0,08)$ & $4(0,09)$ & $14(0,08)$ \\
\hline Uso pasado/no uso & $12.349(99,48)$ & $4.413(99,30)$ & $16.762(99,43)$ \\
\hline \multicolumn{4}{|c|}{ Interacciones potenciales (concomitante) } \\
\hline Antagonistas del calcio & $2.124(17,11)$ & $608(13,68)$ & $2.732(16,21)$ \\
\hline Ciclosporina & $11(0,09)$ & $2(0,05)$ & $13(0,08)$ \\
\hline Inductores CYP3A4 & $4(0,03)$ & $2(0,05)$ & $6(0,04)$ \\
\hline Inhibidores CYP3A4 & $795(6,40)$ & $248(5,58)$ & $1.043(6,19)$ \\
\hline
\end{tabular}

\begin{tabular}{|c|c|c|c|c|c|c|}
\hline \multicolumn{7}{|c|}{$\begin{array}{c}\text { Tabla } 2 \\
\begin{array}{c}\text { Dosis y tipo de estatina utilizada en régimen de terapia intensiva } \\
\text { en el periodo de estudio }\end{array}\end{array}$} \\
\hline \multirow{2}{*}{$\begin{array}{l}\text { Tipo de estatina } \\
\text { (dosis diaria) }\end{array}$} & \multicolumn{5}{|c|}{ Año } & \multirow{2}{*}{$\begin{array}{c}\text { Periodo } \\
\text { 2007-2011 }\end{array}$} \\
\hline & 2007 & 2008 & 2009 & 2010 & 2011 & \\
\hline Atorvastatina $\geq 40 \mathrm{mg}$ & $903(97,73)$ & $954(97,25)$ & $944(93,28)$ & $806(83,52)$ & $451(80,25)$ & $4.058(91,32)$ \\
\hline Fluvastatina $>80 \mathrm{mg}$ & $2(0,22)$ & $2(0,20)$ & $2(0,20)$ & $2(0,21)$ & $0(0,00)$ & $8(0,18)$ \\
\hline Lovastatina $>80 \mathrm{mg}$ & $1(0,11)$ & $2(0,20)$ & $0(0,00)$ & $0(0,00)$ & $0(0,00)$ & $3(0,07)$ \\
\hline Pravastatina $>80 \mathrm{mg}$ & $3(0,32)$ & $1(0,10)$ & $1(0,10)$ & $2(0,21)$ & $0(0,00)$ & $7(0,16)$ \\
\hline Rosuvastatina $>5 \mathrm{mg}$ & - & - & $39(3,85)$ & $140(14,51)$ & $96(17,08)$ & $275(6,19)$ \\
\hline Simvastatina $>40 \mathrm{mg}$ & $15(1,62)$ & $22(2,24)$ & $26(2,57)$ & $13(1,35)$ & $5(0,89)$ & $81(1,82)$ \\
\hline Simvastatina/Ezet $\geq 20 / 10 \mathrm{mg}$ & - & - & - & $2(0,21)$ & $10(1,78)$ & $12(0,27)$ \\
\hline Total terapia intensiva & $924(20,79)$ & $981(22,07)$ & $1.012(22,77)$ & $965(21,71)$ & $565(12,65)$ & $4.444(100,00)$ \\
\hline
\end{tabular}

\begin{tabular}{|c|c|c|c|c|c|c|}
\hline \multicolumn{7}{|c|}{$\begin{array}{c}\text { Tabla } 3 \\
\text { Dosis y tipo de estatina utilizada en régimen de terapia moderada en el periodo de estudio }\end{array}$} \\
\hline \multirow{2}{*}{$\begin{array}{l}\text { Tipo de estatina } \\
\text { (dosis diaria) }\end{array}$} & \multicolumn{5}{|c|}{ Año } & \multirow{2}{*}{$\begin{array}{l}\text { Periodo } \\
2007-2011\end{array}$} \\
\hline & 2007 & 2008 & 2009 & 2010 & 2011 & \\
\hline Atorvastatina $<40 \mathrm{mg}$ & $1.020(28,57)$ & $827(26,68)$ & $782(28,72)$ & $512(27,23)$ & $312(27,40)$ & $3.368(27,81)$ \\
\hline Fluvastatina $\leq 80 \mathrm{mg}$ & $216(6,05)$ & $147(4,74)$ & $110(4,04)$ & $52(2,77)$ & $6(0,53)$ & $531(4,28)$ \\
\hline Lovastatina $\leq 80 \mathrm{mg}$ & $75(2,10)$ & $44(1,42)$ & $30(1,10)$ & $20(1,06)$ & $2(0,18)$ & $171(1,38)$ \\
\hline Pitavastatina $\leq 4 \mathrm{mg}$ & - & - & - & - & $19(1,67)$ & $19(0,15)$ \\
\hline Pravastatina $\leq 80 \mathrm{mg}$ & $370(10,36)$ & $262(8,45)$ & $139(5,10)$ & $88(4,68)$ & $51(4$, & $910(7,33)$ \\
\hline Rosuvastatina $\leq 5 \mathrm{mg}$ & - & - & $9(0,33)$ & $37(1,97)$ & $26(2,28)$ & $72(0,58)$ \\
\hline Simvastatina $\leq 40 \mathrm{mg}$ & $1.889(52,91)$ & $1.820(58,71)$ & $1.654(60,72)$ & $1.171(62,29)$ & $723(63,48)$ & $7.257(58,46)$ \\
\hline Total terapia moderada & $3.570(28,76)$ & $3.100(24,97)$ & $2.724(21,94)$ & $1.880(15,15)$ & $1.139(9,18)$ & $12.413(100,00)$ \\
\hline
\end{tabular}




\begin{tabular}{|c|c|c|c|c|}
\hline \multicolumn{5}{|c|}{\begin{tabular}{|c|} 
Tabla 4 \\
Modelos de regresión logística multivariante: \\
factores asociados al inicio con terapia intensiva con estatinas
\end{tabular}} \\
\hline & \multicolumn{2}{|c|}{ Modelo A } & \multicolumn{2}{|c|}{ Modelo B } \\
\hline & OR & IC95\% & OR & IC95\% \\
\hline \multicolumn{5}{|l|}{ Edad (años) } \\
\hline $45-64$ & referencia & & referencia & \\
\hline $65-74$ & 0,98 & $0,80-1,19$ & 0,90 & $0,80-1,01$ \\
\hline $75-101$ & 1,39 & $1,15-1,69$ & 1,22 & $1,09-1,37$ \\
\hline Sexo (hombre) & 1,70 & $1,44-2,00$ & 1,73 & $1,57-1,90$ \\
\hline $\begin{array}{l}\text { Enfermedad isquémica } \\
\text { coronaria }\end{array}$ & 1,71 & $1,44-2,04$ & 1,54 & $1,34-1,76$ \\
\hline Accidente cerebrovascular & - & - & 1,20 & $1,05-1,37$ \\
\hline Accidente isquémico transitorio & 1,24 & $0,97-1,59$ & 1,22 & $1,05-1,42$ \\
\hline Enfermedad arterial periférica & 0,71 & $0,55-0,91$ & 0,68 & $0,57-0,80$ \\
\hline \multicolumn{5}{|l|}{ Año de prescripción de la estatina } \\
\hline 2007 & referencia & & referencia & \\
\hline 2008 & 1,41 & $1,11-1,78$ & 1,19 & $1,04-1,36$ \\
\hline 2009 & 1,59 & $1,26-2,01$ & 1,43 & $1,25-1,63$ \\
\hline 2010 & 1,99 & $1,56-2,54$ & 1,95 & $1,70-2,23$ \\
\hline 2011 & 1,98 & $1,49-2,63$ & 1,82 & $1,55-2,14$ \\
\hline Fumador & 1,62 & $1,34-1,95$ & 1,50 & $1,35-1,67$ \\
\hline Hipertensión (tratada) & 1,41 & $1,20-1,65$ & 1,21 & $1,10-1,32$ \\
\hline Proteinuria & 0,46 & $0,22-0,95$ & - & - \\
\hline Insuficiencia renal & - & - & 0,79 & $0,65-0,97$ \\
\hline \multicolumn{5}{|l|}{ Uso de hipolipemiantes } \\
\hline \multicolumn{5}{|l|}{ Ezetimiba } \\
\hline - uso pasado/no uso & referencia & & referencia & \\
\hline - uso reciente & - & - & 0,45 & $0,05-3,81$ \\
\hline - uso concomitante & - & - & 1,84 & $1,08-3,15$ \\
\hline \multicolumn{5}{|l|}{ Fibratos } \\
\hline - uso pasado/no uso & referencia & & referencia & \\
\hline - uso reciente & 2,32 & $1,27-4,26$ & & \\
\hline - uso concomitante & 1,00 & $0,60-1,66$ & & \\
\hline \multicolumn{5}{|l|}{ Interacciones } \\
\hline Antagonistas del calcio & 0,77 & $0,63-0,95$ & 0,78 & $0,69-0,88$ \\
\hline Inductores enzimáticos & - & - & 8,59 & $0,76-96,92$ \\
\hline
\end{tabular}

OR: odds ratio; IC95\%: intervalo de confianza del 95\%.

a Modelo A (principal): $\mathrm{n}=3.987$. $\mathrm{P}$ (Hosmer-Lemeshow): 0,09 variables no significativas con $\mathrm{p}<0,05$ para entrada y $\mathrm{p} \geq 0,10$ para salida, $A C V$, insuficiencia renal, insuficiencia cardiaca, hipotiroidismo, diabetes mellitus (tratada), IMC, alcoholismo, niveles de cLDL, CT elevado, bajo HDL, triglicéridos elevados, uso de ezetimiba, uso de "otros hipolipemiantes", inhibidores del CYP3A4. Los inductores enzimáticos y ciclosporina no pudieron ser evaluados por colinearidad del modelo.

b Modelo B (Sin valores lipídicos): $\mathrm{n}=11.200$. P(Hosmer-Lemeshow): 0,52 variables no significativas con $\mathrm{p}<0,05$ para entrada $\mathrm{y} p \geq 0,10$ para salida: diabetes mellitus (tratada), proteinuria, IMC, hipotiroidismo, insuficiencia cardiaca, alcoholismo, uso de fibratos, uso de "otros hipolipemiantess", inhibidores del CYP3A4, ciclosporina.

En términos relativos, la utilización de terapia intensiva respecto al uso de la moderada aumentó durante el periodo de estudio, pasando del 20,6\% en el año 2007 al 33,0\% en 2011.

La tabla 4 muestra los resultados de los modelos de regresión logística con los factores que se asociaron al inicio con terapia intensiva respecto a la moderada. El análisis univariante se presenta en la tabla 5. Los predictores para el inicio de terapia intensiva identificados en el modelo principal fueron el año de prescripción, ser hombre (OR ajustada: 1,70; IC95\%: 1,44-2,00), tener más de 75 años $(1,39 ; 1,15-1,69)$, tener historia previa de enfermedad isquémica coronaria $(1,71$; 


\begin{tabular}{|c|c|c|}
\hline \multicolumn{3}{|c|}{$\begin{array}{c}\text { Tabla } 5 \\
\text { Análisis de regresión logística univariante: } \\
\text { factores asociados al inicio con terapia } \\
\text { intensiva con estatinas }\end{array}$} \\
\hline Variable & Odds ratio & IC95\% \\
\hline \multicolumn{3}{|l|}{ Edad en años } \\
\hline $45-64$ & Referencia & \\
\hline $65-74$ & 0,73 & $0,67-0,80$ \\
\hline $75-110$ & 0,81 & $0,75-0,88$ \\
\hline Sexo (hombre) & 1,73 & $1,61-1,87$ \\
\hline Enfermedad isquémica coronaria & 1,38 & $1,28-1,48$ \\
\hline \multicolumn{3}{|l|}{ IMC } \\
\hline$\leq 25$ & 0,97 & $0,86-1,08$ \\
\hline $25,1-29,9$ & Referencia & \\
\hline $30-39,9$ & 1,02 & $0,93-1,12$ \\
\hline Fumador & 1,56 & $1,43-1,71$ \\
\hline Consumo elevad & 1,18 & $1,04-1,34$ \\
\hline Accidente cerebr & 0,95 & $0,88-1,02$ \\
\hline Accidente isquémico transitorio & 0,96 & $0,87-1,07$ \\
\hline Enfermedad arterial periférica & 0,61 & $0,55-0,68$ \\
\hline Hipertensión tratada & 0,99 & $0,93-1,06$ \\
\hline Diabetes mellitus tratada & 0,88 & $0,81-0,96$ \\
\hline Hipotiroidismo & 0,69 & $0,59-0,81$ \\
\hline CT elevado & 0,74 & $0,65-0,84$ \\
\hline \multicolumn{3}{|l|}{ cLDL(mg/dl) } \\
\hline$<130$ & Referencia & \\
\hline $130-159,9$ & 0,84 & $0,73-0,97$ \\
\hline $160-189,9$ & 0,87 & $0,74-1,02$ \\
\hline$\geq 190$ & 0,56 & $0,43-0,74$ \\
\hline Triglicérido & 1,02 & $0,89-1,15$ \\
\hline Bajo HDL & 1,12 & $0,98-1,27$ \\
\hline \multicolumn{3}{|l|}{ Año de prescripción de la estatina } \\
\hline 2007 & Referencia & \\
\hline 2008 & 1,22 & $1,10-1,35$ \\
\hline 2009 & 1,44 & $1,30-1,59$ \\
\hline 2010 & 1,98 & $1,78-2,21$ \\
\hline 2011 & 1,91 & $1,68-2,16$ \\
\hline Hipertensión tratada & 0,99 & $0,93-1,06$ \\
\hline Proteinuria & 0,80 & $0,58-1,12$ \\
\hline Insuficiencia renal & 0,76 & $0,65-0,90$ \\
\hline \multicolumn{3}{|l|}{ Uso de hipolipemiantes } \\
\hline \multicolumn{3}{|l|}{ Ezetimiba } \\
\hline - uso pasado/no uso & Referencia & \\
\hline - uso reciente & 0,25 & $0,03-1,97$ \\
\hline - uso concomitante & 1,75 & $1,19-2,56$ \\
\hline \multicolumn{3}{|l|}{ Fibratos } \\
\hline - uso pasado/no uso & Referencia & \\
\hline - uso reciente & 1,24 & $0,84-1,82$ \\
\hline - uso concomitante & 1,16 & $0,91-1,48$ \\
\hline \multicolumn{3}{|l|}{ Otros } \\
\hline - uso pasado/no uso & Referencia & \\
\hline - uso reciente & 1,12 & $0,35-3,57$ \\
\hline - uso concomitante & 1,40 & $0,88-2,22$ \\
\hline \multicolumn{3}{|l|}{ Interacciones } \\
\hline Antagonistas del calcio & 0,77 & $0,70-0,85$ \\
\hline Inductores enzimáticos & 1,40 & $0,26-7,63$ \\
\hline Inhibidores enzimáticos & 0,86 & $0,75-1,00$ \\
\hline Ciclosporina & 0,51 & $0,11-2,29$ \\
\hline
\end{tabular}

1,44-2,04) o de AIT $(1,24 ; 0,97-1,59)$, tabaquismo $(1,62 ; 1,34-1,95)$, hipertensión tratada $(1,41 ; 1,20-1,65)$ y tratamiento previo con fibratos $(2,32 ; 1,27-4,26)$.

Los predictores que encontró el modelo principal fueron similares al modelo sin valores lipídicos (Modelo B). Sin embargo, en éste último se encontraron como predictores adicionales del inicio con terapia intensiva la historia previa de ACV (OR ajustada: 1,20; IC95\%:1,05-1,37), y el uso concomitante de ezetimiba $(1,84 ; 1,08-3,15)$.

Los modelos mostraron una moderada bondad de ajuste que fue mayor en el principal y ambos mostraron una discreta capacidad discriminativa (C-estadístico: 0,66 en el modelo principal y 0,64 en el modelo sin valores lipídicos).

\section{DISCUSIÓN}

En este estudio con más de 16 mil pacientes con antecedentes de enfermedad vascular y/o cardiaca atendidos ambulatoriamente, el tipo de acontecimiento vascular fue determinante para iniciar la utilización de terapia intensiva. Así, la historia previa de cardiopatía isquémica o accidente cerebral isquémico transitorio, se asociaron con el inicio del tratamiento con terapia intensiva, mientras que la historia previa de enfermedad arterial periférica se asoció con la utilización de terapia moderada. La proporción de hipercolesterolemia al inicio del tratamiento con estatinas para la prevención secundaria fue mayor que la estimada para la población general española $(50,5 \%)^{19}$. En distintas guías de práctica clínica se recomienda el inicio del tratamiento con terapia intensiva (o dosis altas) en los pacientes con cardiopatía isquémica y síndrome coronario agudo $\mathrm{o}^{1,7,16,20} \mathrm{o}$ antes del procedimiento de intervención coronaria percutánea ${ }^{5}$, y la mayor parte de los ensayos clínicos realizados con terapia intensiva se han llevado a cabo en pacientes con historia previa de enfermedad coronaria, IAM o angina inestable $e^{4,8,21,22}$. La terapia intensiva ha demostrado reducir la mortalidad por todas las causas en pacientes con síndrome coronario agudo $^{9,18}$. Sin embargo, en distintos ensayos 
realizados en pacientes con historia previa de cardiopatía isquémica o IAM, la mortalidad por todas las causas no tuvo diferencias estadísticamente significativas en los sujetos tratados con terapia intensiva respecto a los tratados con terapia moderada $a^{4,8,22}$.

En el ensayo The Stroke Prevention by Aggressive Reduction in Cholesterol Levels (SPARCL), que incluía pacientes con antecedentes de enfermedad cerebrovascular tratados con atorvastatina $80 \mathrm{mg}$ respecto a placebo, se observó un efecto protector de la terapia intensiva para los ictus de naturaleza isquémica, pero por el contrario se asociaba a mayor riesgo de ictus de tipo hemorrágico ${ }^{23}$. Diversos estudios epidemiológicos sugieren una asociación entre niveles bajos de colesterol y riesgo de hemorragia cerebral ${ }^{24}$. En nuestro estudio el inicio de terapia intensiva con estatinas se asoció con historia previa de AIT.

El tabaquismo y la hipertensión se asociaron con la terapia intensiva, sin embargo no se hallaron diferencias estadísticamente significativas para el inicio de terapia intensiva en función del IMC o la diabetes (tratada con insulina o antidiabéticos orales).

La utilización de terapia intensiva se asoció con ser mayor de 75 años en comparación con la mediana edad (45/64 años). Existe controversia sobre el comienzo de tratamiento con estatinas en pacientes de edades muy $\operatorname{avanzadas}^{25}$ y algunos estudios encuentran una asociación inversa entre los niveles lipídicos y en la enfermedad cardiaca en pacientes de más 80 años de edad ${ }^{26}$. Un metaanálisis de cinco ensayos clínicos aleatorizados que comparaban terapia intensiva con la moderada en pacientes con enfermedad coronaria de 65 a 85 años, encontró reducción de infarto (no mortal) e ictus pero no halló diferencias en la mortalidad cardiovascular o en la mortalidad por todas las causas ${ }^{27}$. Por otra parte, la generalización de los resultados en personas muy ancianas a partir de los ensayos clínicos llevados a cabo hasta la fecha con terapia intensiva es cuestionable, porque la mayoría excluyeron la participación de mayores de 80 años $^{4,8,23}$. De acuerdo con un estudio de cohortes retrospectivo con 18.311 pacientes ancianos (edad media 77) que padecían síndrome coronario agudo, la terapia intensiva no ofrecería un beneficio adicional (en términos de una disminución de las rehospitalizaciones o la mortalidad) respecto a la terapia modera$\mathrm{da}^{27}$. En la última actualización de las guías de práctica clínica estadounidenses no se recomienda el tratamiento con terapia intensiva en pacientes con edades muy avanzadas ${ }^{6}$.

Al igual que en otros estudios ${ }^{11,29,30}$, la fecha de prescripción de la estatina fue un predictor importante de la probabilidad para la prescripción de terapia intensiva. Esto podría deberse a la fecha de publicación de los principales ensayos clínicos realizados con terapia intensiva o al abaratamiento del uso de dosis altas que ha supuesto la aparición de genéri$\cos ^{2}$. No obstante, cabe destacar que al inicio del periodo de estudio, la atorvastatina suponía el 98\% de las prescripciones en el grupo de terapia intensiva pero, paradójicamente, se ha sustituido en parte por rosuvastatina, que no dispone de genérico (comercializada a finales del año 2008) y que ha supuesto el 17\% de las prescripciones de terapia intensiva en 2011.

La enfermedad arterial periférica se asoció con el uso de dosis moderadas. Ya en el ensayo clínico del Heart Protection Study Collaborative Group, llevado a cabo con terapia moderada (simvastatina $40 \mathrm{mg}$ ) respecto a placebo en pacientes con antecedentes de enfermedad arterial periférica, se encontró beneficio clínico ${ }^{31}$. Cabe destacar que en las guías europeas para la prevención de la enfermedad cardiovascular 2012 se recomienda (clase I nivel de evidencia A) tratar a los pacientes con enfermedad arterial periférica como si fuesen pacientes de alto riesgo cardiovascular, ya que se considera una condición con riesgo equivalente al de la enfermedad coronaria ${ }^{16}$. En este caso la interpretación de la evidencia procedente de los ensayos clínicos que comparaban terapia intensiva 
respecto a la terapia moderada ${ }^{4,22}$ puede verse limitada por el menor porcentaje de pacientes con historia previa de enfermedad arterial periférica incluidos en los estudios, y/o la potencia estadística para encontrar diferencias ${ }^{32}$.

En este estudio la proteinuria se asoció al uso de terapia moderada (protección frente a la terapia intensiva). El uso de estatinas potentes como rosuvastatina se ha asociado con el aumento del riesgo de proteinuria en dos ensayos clínicos ${ }^{33}$. Por otra parte, recientemente se ha encontrado un aumento de riesgo de daño renal agudo en pacientes tratados con altas dosis de estatina respecto a los tratados con dosis bajas en un estudio llevado a cabo con distintas bases de datos ${ }^{34}$.

Por último, no se hallaron diferencias estadísticamente significativas respecto al uso de terapia moderada o intensiva en función de los niveles de lípidos, a diferencia de otro estudio similar de utilización de estatinas en el que los valores elevados de cLDL se asociaron con el inicio de la terapia intensiva para la prevención primaria de enfermedad cardiovascular ${ }^{11}$. Una de las características de la terapia intensiva es la disminución abrupta de los niveles de cLDL $^{35}$. Sin embargo, en pacientes con enfermedad coronaria establecida, la terapia intensiva ha demostrado ser más beneficiosa que la terapia moderada en la prevención de infarto con independencia de los niveles de $\mathrm{cLDL}^{10}$. Las guías europeas de práctica clínica recomiendan el inicio del tratamiento con estatinas con independencia de la cifra basal de cLDL para los pacientes con cardiopatía isquémica ${ }^{5}$ y en la última actualización de la guía estadounidense la selección de la terapia intensiva en pacientes con enfermedad cardiovascular preexistente sería también independiente del nivel basal de cLDL $^{6}$.

Este estudio no está exento de limitaciones potenciales. En primer lugar, el número de valores de laboratorio válidos para el análisis condicionó la potencia estadística del modelo principal y, por otra parte, la población con valores de test de laborato- rio disponibles podría ser entendida como una muestra altamente seleccionada con un comportamiento diferente al resto. Con el fin de examinar este aspecto, se llevó a cabo un análisis adicional con un modelo sin los valores de laboratorio de lípidos pero con una mayor muestra poblacional (Modelo B), siendo los resultados similares a los del análisis para el modelo principal (Modelo A). En segundo lugar, como en toda base de datos de atención primaria siempre pueden existir limitaciones debidas a una recogida incompleta de datos, prescripciones o información no recogida sobre factores de confusión, etcétera. En particular, no existía información sobre estilos de vida como dieta o actividad física, historia familiar de enfermedad cardiovascular o presencia de dislipemias genéticas que podrían influir en el patrón de uso de estos medicamentos.

Para minimizar el posible sesgo en los valores de lípidos en el caso de prescripciones de estatinas no registradas durante el ingreso hospitalario (ámbito perioperatorio) se tuvieron en cuenta los valores lipídicos registrados hasta el mes previo a la fecha índice.

Por último, se desconocen las recomendaciones concretas que se siguieron para la prescripción de estatinas y/o la posible adherencia a las guías de práctica clínica ${ }^{36,37}$, no pudiendo descartarse totalmente el sesgo de indicación.

Sin embargo, a fin de minimizar posibles sesgos, los resultados se ajustaron en función de variables relacionadas con la prescripción de estatinas, incluyendo comorbilidades que pudieran causar dislipemia secundaria, como el hipotiroidismo, o contraindicaciones y potenciales interacciones con otros medicamentos.

Uno de cada cuatro pacientes inició el tratamiento con estatinas como prevención secundaria de enfermedad vascular utilizando terapia intensiva. El uso de la terapia intensiva ha aumentado en los últimos años lo que ha estado determinado por el tipo de evento vascular previo. Otros factores como ser varón, fumador o hipertenso también se asociaron con el uso de terapia 
intensiva. Sin embargo, no se encontraron diferencias estadísticamente significativas con respecto a la prescripción de terapia intensiva o moderada en función del valor del cLDL. Tampoco se hallaron diferencias significativas en la presencia de contraindicaciones, uso concomitante de fibratos u otros medicamentos que podrían interaccionar en mayor medida con la terapia intensiva (por ejemplo inhibidores CYP3A4). Sin embargo, la terapia intensiva se asoció más con ser mayor de 75 años, sujetos en los que el balance beneficio riesgo podría ser discutible.

\section{AGRADECIMIENTOS}

Los autores agradecen la excelente colaboración de los Médicos participantes en la Base de datos para la Investigación Farmacoepidemiológica en Atención Primaria (BIFAP).

\section{BIBLIOGRAFÍA}

1. Maiques Galán A, Brotons Cuixart C, Villar Álvarez F, Navarro Pérez J, Lobos-Bejarano JM, Ortega Sánchez-Pinilla R, et al. Recomendaciones preventivas cardiovasculares. Aten Primaria. 2012;44 Supl 1:3-15.

2. Catalá-López F, Sanfélix-Gimeno G, Ridao M, Peiró $\mathrm{S}$. When are statins cost-effective in cardiovascular prevention? A systematic review of sponsorship bias and conclusions in economic evaluations of statins. PLoS One. 2013;8:e69462.

3. Agencia Española de Medicamentos y Productos Sanitarios. Observatorio de uso de medicamentos. Utilización de medicamentos hipolipemiantes en España durante el periodo 2000-2012. Madrid: Agencia Española de Medicamentos y Productos Sanitarios; 2013. [Citado 1 septiembre 2014]. Disponible en: http:// www.aemps.gob.es/gl/medicamentosUsoHumano/observatorio/docs/hipolipemiantes-2000-2012.pdf

4. LaRosa JC, Grundy SM, Waters DD, Shear C, Barter P, Fruchart JC, et al. Intensive lipid lowering with atorvastatin in patients with stable coronary disease. $\mathrm{N}$ Engl J Med. 2005;352:1425-35.

5. Grupo de Trabajo de la Sociedad Europea de Cardiología sobre diagnóstico y tratamiento de la cardiopatía isquémica estable. Guía de Práctica Clínica de la ESC 2013 sobre diagnóstico y tratamiento de la cardiopatía isquémica estable. Rev Esp Cardiol. 2014;67:135. e1-e81.
6. Stone NJ, Robinson J, Lichtenstein AH, Merz CN, Blum CB, Eckel RH, et al. 2013 ACC/AHA Guideline on the Treatment of Blood Cholesterol to Reduce Atherosclerotic Cardiovascular Risk in Adults: A Report of the American College of Cardiology/American Heart Association Task Force on Practice Guidelines. Circulation. J Am Coll Cardiol. 2013; S0735-1097:06028-2.

7. National Institute for Health and Clinical Excellence. Lipid modification. Cardiovascular risk assessment and the modification of blood lipids for the primary and secondary prevention of cardiovascular disease. NICE Clinical guideline 181. London: NICE; 2014. [Citado 1 febrero 2015]. Disponible en: http://www.nice.org. uk/guidance/cg181/resources/guidance-lipid-modification-cardiovascular-risk-assessment-and-the-modification-of-blood-lipids-for-the-primary-and-secondaryprevention-of-cardiovascular-disease-pdf

8. Armitage J, Bowman L, Wallendszus K, Bulbulia R, Rahimi K, Haynes R, et al. Intensive lowering of LDL cholesterol with $80 \mathrm{mg}$ versus $20 \mathrm{mg}$ simvastatin daily in 12,064 survivors of myocardial infarction: a doubleblind randomised trial. Study of the Effectiveness of Additional Reductions in Cholesterol and Homocysteine (SEARCH). Lancet. 2010;376:1658-69.

9. Silva M, Matthews ML, Jarvis C, Nolan NM, Belliveau P, Malloy M, et al. Meta-analysis of drug-induced adverse events associated with intensive-dose statin therapy. Clin Ther. 2007;29:253-60.

10. Josan K, Majumdar SR, McAlister FA. The efficacy and safety of intensive statin therapy: a meta-analysis of randomized trials. CMAJ. 2008;178:576-84.

11. Macías Saint-Gerons D, de la Fuente Honrubia C, Montero Corominas D, Gil MJ, de Andrés-Trelles F, Catalá-López F. Standard and intensive lipid-lowering therapy with statins for the primary prevention of vascular diseases: a population-based study. Eur J Clin Pharmacol. 2014;70:99-108.

12. Molnar AO, Coca SG, Devereaux PJ, Jain AK, Kitchlu A, Luo J, et al. Statin use associates with a lower incidence of acute kidney injury after major elective surgery. J Am Soc Nephrol. 2011;22(5):939-46.13. Food and Drug Administration. FDA Drug safety communication: new restrictions, contraindications, and dose limitations for Zocor (simvastatin) to reduce the risk of muscle injury. 2011 [Citado 1 Septiembre 2014]. Disponible en http://www.fda.gov/Drugs/DrugSafety/ ucm256581.htm

14. National Cholesterol Education Program (NCEP) Expert Panel on Detection, Evaluation, and Treatment of High Blood Cholesterol in Adults (Adult Treatment Panel III). Third report of the National Cholesterol Education Program (NCEP) Expert Panel on Detection, Evaluation, and Treatment of High Blood Cholesterol in Adults (Adult Treatment Panel III) final report. Circulation. 2002;106:3143-3421. 
15. Miller M, Stone NJ, Ballantyne C, Bittner V, Criqui $\mathrm{MH}$, Ginsberg HN, et al. Triglycerides and cardiovascular disease: a scientific statement from the American Heart Association Circulation. 2011;123:2292-333.

16. European Guidelines on cardiovascular disease prevention in clinical practice (version 2012): the Fifth Joint Task Force of the European Society of Cardiology and Other Societies on Cardiovascular Disease Prevention in Clinical Practice (constituted by representatives of nine societies and by invited experts). Fifth Joint Task Force of the European Society of Cardiology1, European Association of Echocardiography, European Association of Percutaneous Cardiovascular Interventions, European Heart Rhythm Association, Heart Failure Association, European Association for Cardiovascular Prevention \& Rehabilitation et al. Eur J Prev Cardiol. 2012 Aug;19(4):585-667.

17. Golomb BA, Evans MA. Statin adverse effects: a review of the literature and evidence for a mitochondrial mechanism. Am J Cardiovasc Drugs. 2008;8:373418 .

18. Mostaza JM, Lahoz C, Morales-Olivas F, Pinto X, Tranche S, Suarez-Tembra et al. Riesgo de interacciones farmacológicas por la coadministración de estatinas con fármacos metabolizados por la isoenzima $3 \mathrm{~A} 4$ del citocromo P450: estudio epidemiológico, transversal y multicéntrico. Med Clin (Barc). 2014 Nov 18;143(10):427-32.

19. Guallar-Castillón P, Gil-Montero M, León-Muñoz LM, Graciani A, Bayán-Bravo A, Taboada JM, et al. Magnitud y manejo de la hipercolesterolemia en la población adulta de España, 2008-2010, el estudio ENRICA. Rev Esp Cardiol 2012; 65(6):551-8

20. SIGN (Scottish Intercollegiate Guidelines Network). Risk estimation and the prevention of cardiovascular disease. Guideline No 97. Edinburgh: SIGN, 2007. [Citado 1 septiembre 2014]. Disponible en: http://www.sign.ac.uk/ pdf/sign 97.pdf

21. De Lemos JA, Blazing MA, Wiviott SD, Lewis EF, Fox KA, White HD, et al. Early intensive vs a delayed conservative simvastatin strategy in patients with acute coronary syndromes: phase $\mathrm{Z}$ of the $\mathrm{A}$ to $\mathrm{Z}$ trial. JAMA. 2004;292:1307-16.

22.. Pedersen TR, Faergeman O, Kastelein JJ, Olsson AG, Tikkanen MJ, Holme I, et al. High-dose atorvastatin vs usual-dose simvastatin for secondary prevention after myocardial infarction: the IDEAL study: a randomized controlled trial. JAMA. 2005;294:2437-45.

23. Amarenco P, Bogousslavsky J, Callahan A 3rd, Goldstein LB, Hennerici M, Rudolph AE, et al. High-dose atorvastatin after stroke or transient ischemic attack. N Engl J Med. 2006;355:549-59.
24. Lee SH, Bae HJ, Yoon BW, Kim H, Kim DE, Roh JK. Low concentration of serum total cholesterol is associated with multifocal signal loss lesions on gradient-echo magnetic resonance imaging: analysis of risk factors for multifocal signal loss lesions. Stroke. 2002; 33:2845-9.

25. Desai DA, Zakaria S, Ouyang P. Initiation of statin therapy: are there age limits? Curr Atheroscler Rep. 2012; $14: 17-25$

26. Savoie I, Kazanjian A. Utilization of lipid-lowering drugs in men and women a reflection of the research evidence? J Clin Epidemiol. 2002;55:95-101.

27. Yan YL, Qiu B, Hu LJ, Jing XD, Liu YJ, Deng SB et al. Efficacy and safety evaluation of intensive statin therapy in older patients with coronary heart disease: a systematic review and meta-analysis. Eur J Clin Pharmacol. 2013;69: 2001-9.

28. Choudhry NK, Levin R, Winkelmayer WC. Statins in elderly patients with acute coronary syndrome: an analysis of dose and class effects in typical practice. Heart. 2007;93:945-51

29. Macías Saint-Gerons D, de la Fuente Honrubia C, de Andrés-Trelles F, Catalá-López F. Prevención primaria de las enfermedades cardiovasculares con estatinas en pacientes con diabetes. Med Clin (Barc). 2014. pii: S00257753(14)00341-8. doi: 10.1016/j.medcli.2014.05.001

30. Javed U, Deedwania PC, Bhatt DL, Cannon CP, Dai D, Hernandez AF, et al. Use of intensive lipid-lowering therapy in patients hospitalized with acute coronary syndrome: an analysis of 65,396 hospitalizations from 344 hospitals participating in Get With The Guidelines (GWTG). Am Heart J. 2010;160:1130-6.

31. Heart Protection Study Collaborative Group. Randomized trial of the effects of cholesterol-lowering with simvastatin on peripheral vascular and other major vascular outcomes in 20,536 people with peripheral arterial disease and other high-risk conditions. J Vasc Surg. 2007;45:645-654;

32. Mohler ER 3rd, Hiatt WR, Creager MA. Cholesterol reduction with atorvastatin improves walking distance in patients with peripheral arterial disease. Circulation. 2003;108:1481-6.

33. Kostapanos MS, Milionis HJ, Elisaf MS. Rosuvastatin-associated adverse effects and drug-drug interactions in the clinical setting of dyslipidemia. Am J Cardiovasc Drugs. 2010;10:11-28.

34. Dormuth CR, Hemmelgarn BR, Paterson JM, James MT, Teare GF, Raymond CB, et al. Use of high potency statins and rates of admission for acute kidney injury: multicenter, retrospective observational analysis of administrative databases. BMJ. 2013; 346:f880. 
35. Cholesterol Treatment Trialists' (CTT) Collaboration, Baigent C, Blackwell L, Emberson J, Holland LE, Reith C, et al. Efficacy and safety of more intensive lowering of LDL cholesterol: a meta-analysis of data from 170,000 participants in 26 randomised trials. Lancet. $2010 ; 376: 1670-81$.

36. Royo-Bordonada MÁ, Lobos JM, Brotons C, Villar F, de Pablo C, Armario P, et al. El estado de la prevención cardiovascular en España. Med Clin (Barc). 2014; 142(1):7-14. doi: 10.1016/j.medcli.2012.09.046.

37. Sanfélix-Gimeno G, Peiró S, Librero J, AusejoSegura M, Suárez-Alemán C, Molina-López T, et al; Grupo IUM-SNS. Análisis poblacional por áreas de salud de las variaciones en consumo, precio y gasto de medicamentos cardiovasculares en 8 comunidades autónomas, España, 2005. Rev Esp Salud Publica. 2010 Jul-Aug;84(4):389-407. 